\title{
Low Cost Microcontroller Based Decay Measurement System for Display Phosphors
}

\author{
V. V. Rangari ${ }^{1}$, A. P. Bhat ${ }^{2}$, V. A. Joshi ${ }^{3}$, S. J. Dhoble ${ }^{1, *}$ \\ ${ }^{1}$ Department of Physics, R.T.M. Nagpur University, Nagpur - 440033 India \\ ${ }^{2}$ Depatrment of Electronics, R.T.M. Nagpur University Nagpur - 440033 India \\ ${ }^{3}$ Department of Instrumentation, V.P.M’s. Polytechnic, Thane, India \\ *Corresponding author: anup_b5@yahoo.com
}

Received October 15, 2014; Revised November 01, 2014; Accepted December 23, 2014

\begin{abstract}
An attempt is made to design and develop a low cost, simple but efficient instrument to measure the decay time of luminescence in phosphors used in displays. The approach employed here is generally an embedded system designed around an intelligent microcontroller Atmega-16 of Atmale. The system is using a time domain technique of measurement and is further enhanced by developing necessary software for online-offline analysis.
\end{abstract}

Keywords: low cost, luminacencence, embedded sysytem, time doaim analysis, standalone system

Cite This Article: V. V. Rangari, A. P. Bhat, V. A. Joshi, and S. J. Dhoble, "Low Cost Microcontroller Based Decay Measurement System for Display Phosphors." American Journal of Electrical and Electronic Engineering, vol. 2, no. 6 (2014): 180-184. doi: 10.12691/ajeee-2-6-5.

\section{Introduction}

Large flat-panel information displays or home cinemas, including rapidly developed 3D (three dimensional) devices, are characteristic examples of utilizing and commercializing the rapidly developing advances in the technology of plasma display panels (PDPs) in our daily life $[1,2]$. Plasma better satisfies the needs of high-quality $3 \mathrm{D}$ displays than LCD/LED. The main reason is that it provides much faster refresh rate, which is an important feature in suppressing lagging or blurring effects. Moreover, the best true resolution of an LCD/LED TV screen can achieve a (maximum) display of 700 or 800 lines at $240 \mathrm{~Hz}$, whereas a plasma screen can achieve a display of 1080 lines at $600 \mathrm{~Hz}$. The excitation source of PDPs is actually the result of the discharge of a noble gas in vacuum, which occurs in the ultra- violet region of the light spectrum. For instance, the discharge of Xe emits photons with wavelengths at $147 \mathrm{~nm}$ and $172 \mathrm{~nm}$. Thus, phosphors have emerged as materials of particular interest for light conversion. In other words, the quality of PDPs strongly depends on the quality of phosphors. Suitable phosphors for these applications should feature high efficiency, stability, strong vacuum ultraviolet (VUV) absorption, good colorimetric purity, and proper decay time. With regard to the latter feature, the decay time for 3D PDPs must be extremely short to avoid cross- talk; thus, the decay time of the phosphor should be short enough (within about 2ms) [2,3,4].

PDP phosphors generate light by transitions in activator ions (i.e. $\mathrm{Ce}^{3+}, \mathrm{Tb}^{3+}$ or $\mathrm{Mn}^{2+}$ ). Therefore, phosphor decay time mainly depends on the transition rate of activator. With a low transition rate, phosphors continue to emit light until the most excited activators lose their energy by slow transition. Thus, phosphor decay times are long with low transition rates. Conversely, a fast- decaying phosphor has a high transition rate.

In 3D PDP, satisfactory CIE chromaticity coordinates and decay time of phosphors were attained by adjusting the phosphor mixture. Long lasting phosphors have the decay time in minutes, hours, days etc.

There are two complementary techniques of Decay time measurement: the time domain and the frequency domain. In the time domain, a short pulse of light excites the sample, and the subsequent emission is recorded as a function of time. In the frequency domain, the sample is excited by a modulated source of light. The luminescence emitted by the sample has a similar waveform, but is modulated and phase-shifted from the excitation curve. Both modulation $(M)$ and phase-shift $(\varphi)$ are determined by the decay time of the sample emission; that decay time can be calculated from the observed modulation and phase-shift [5]. In order to measure the decay time of luminescence in prepared phosphors, the instrument was design using time domain technique.

$$
\mathrm{E} 1(\mathrm{~d} 1+\mathrm{X}) 2=\mathrm{E} 2(\mathrm{~d} 2+\mathrm{X}) 2-
$$

$\mathbf{X}$ gives the distance between excitation source and the material holder.

Radiance and Luminance:

Radiance is a measure of the flux density per unit solid viewing angle, expressed in $\mathrm{W} / \mathrm{cm} 2 / \mathrm{sr}$. Radiance is independent of distance for an extended area source, because the sampled area increases with distance, cancelling inverse square losses. The radiance, L, of a diffuse (Lambertian) surface is related to the radiant acceptance (flux density), $\mathrm{M}$, of a surface by the relationship: 


$$
\mathrm{L}=\mathrm{M} / \mathrm{p}
$$

\section{Irradiance from an Extended Source:}

The irradiance, E, at any distance from a uniform extended area source, is related to the radiance, L, of the source by the following relationship, which depends only on the subtended central viewing angle, of the radiance detector:

$$
\mathrm{E}=\mathrm{pL} \sin ^{2}(\mathrm{q} / 2)
$$

\subsection{System Description}

The complete system is constructed using the available components and tools easily available. The block diagram of a system shown in Figure 1.

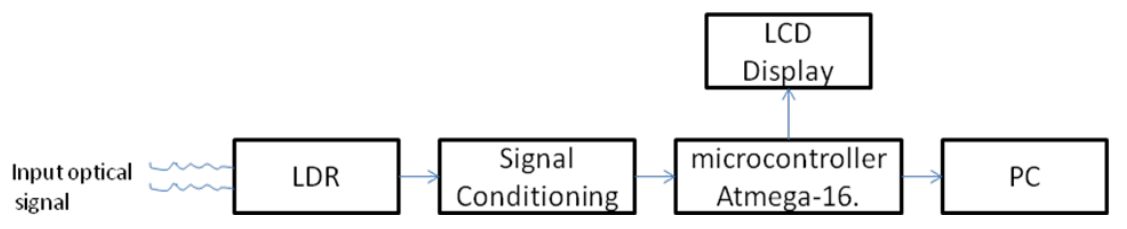

Figure 1. Block diagram of the system

It consists of a light sensing element as LDR. LDR varies the resistance of the material when it exposed to the light, same property of LDR is used for intensity calculation. As the light falls on the LDR surface the resistance of the device is reduce in proportionate with the light fall and produce the appropriate voltage. The voltage getting from the LDR is not at that much high level to drive the direct readout. The voltage from the LDR is amplified with the signal conditioning block, which consist of IC TL 072, single power supply IC. This amplified voltage is feed to the digital world using the microcontroller Atmega-16. The analog voltage is converted using the inbuilt 10-bit resolution ADC acceptable in microcontroller [6]. The microcontroller is programmed in such way that the voltage received at the ADC input is converted into the LUX using inter conversion formula. The voltage converted into the standard string formation is needed by LCD. The analog voltage as well as the converted voltage in lux is shown on LCD screen.

\subsection{Algorithm of the System}

1. First step is to initialize keys, Interrupt vectors panel and LCD Define port B of microcontroller ATmega 16/32 as output port and Port A as Input for ADC.

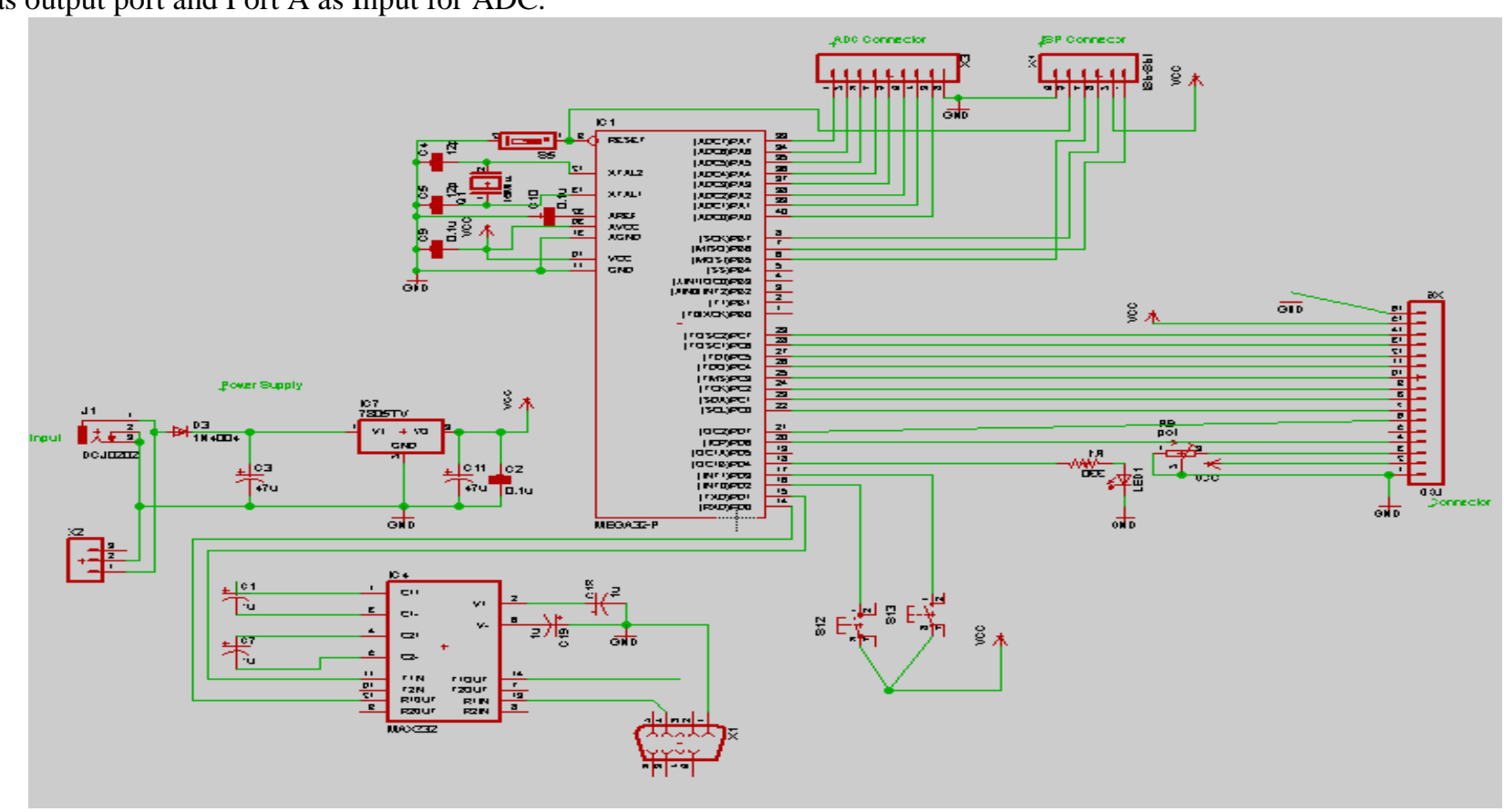

2. Get data through ADC from two different channels of Temperature and Light Intensity continuously after a fixed interval.

3. Value obtained from different channels converted to appropriate form of display.

4. Display the appropriate values of input voltage from LDR and Light Intensity on LCD.

5. Start hardware device to glow a light if sensed light intensity is higher than the set value implying darkness.

6. New value of light intensity can be set using light converted into voltage.

7. New value of transfer rate of data to RS232 is set using keypad as follows.

\section{Design Frame Work}

\subsection{System Design}

The design process is divided into two: Hardware and Software designs.

\subsubsection{Hardware Description}

Figure 2 gives the schematic diagram of the system. Whole circuit diagram is divided into following sections: -

Figure 2. Complete schematic of the system 
(a) Power supply section: The regulated power supplies section made with full wave rectifier (with IN 4007 diodes) using voltage regulator IC 7805 and IC 7812 which provide a constant voltage of $5 \mathrm{~V}$ to the circuit as well as constant $12 \mathrm{~V}$ to relays.

(b) Analog to digital conversion section: Since we have to sense analog parameter i.e. light hence we have to use any analog to digital converter. We have opted for ADC 0809 as it has 8 channels and is microprocessor compatible ADC which is easily available [7]. It will convert the analog signal of the transducer to digital value with respect to the reference voltage, which in our case is $5 \mathrm{~V}$. This reference voltage is obtained using microcontroller Vcc supply voltage, which shifts the reference of ADC as AREF or Vcc voltage reference with output voltage range of 0.1 to $4.88 \mathrm{~V}$ [8].

For the conversion ADC requires a reference frequency which is supplied from inbuilt oscillator available in controller also can be calculated from the crystal oscillator, which work as system clock, in the form of a stable oscillator. The conversion frequency is kept around 1000 $\mathrm{kHz}$. Sensor used for light intensity is LDR. We have considered the reference voltage of ADC as standard for light intensity measurement. In that process $5 \mathrm{~V}$ is considered as full light and $0 \mathrm{~V}$ is considered as darkness. The light intensity is hence displayed in LCD as the reference voltage itself. The voltage scan by ADC is converted into per bit value using the percales, by multiplying 0.00482812 convert the scan voltage into the voltage proportional to light incident. This value is set with bit resolution and reference voltage applied. We used 10 bit ADC with voltage is $5 \mathrm{~V}$ and bit is $1024\left(2^{10}\right)$

(c) Controller section: The analog value is converted to digital value by inbuilt ADC and is picked up by microcontroller Atmega32 that is a 40pin device. The microcontroller is a low-power, high-performance CMOS 16-bit microcomputer with $8 \mathrm{~K}$ bytes of Flash programmable and erasable read only memory (EPROM) [9].

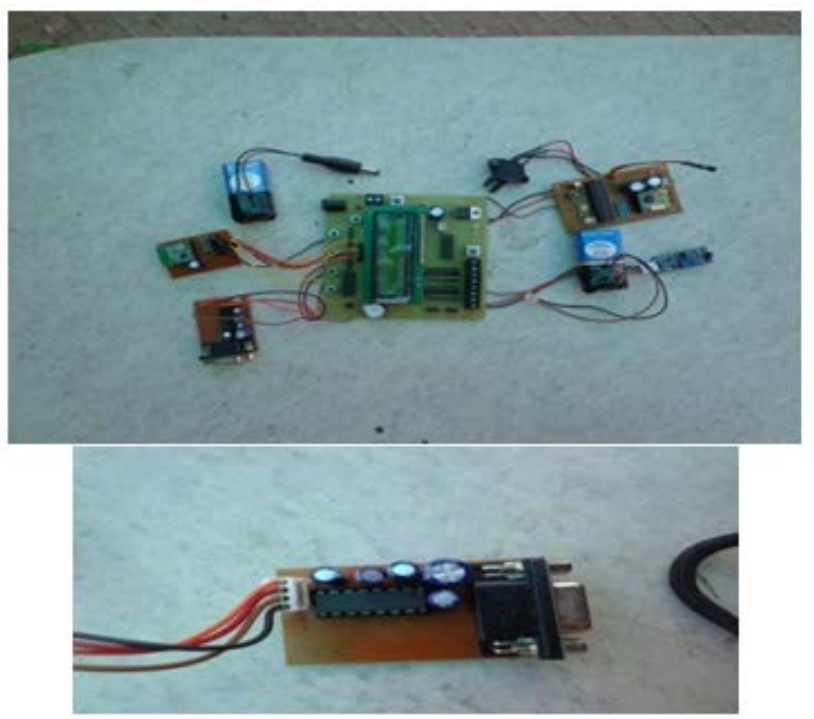

Figure 3. Embedded Control Hardware Circuit with serial communicator RS232

(d) Display section: Since we need to display the data we get from microcontroller a liquid crystal display 44780 LCD is used which is a $2 \times 16$ line display [10,11]. The display shows the voltage receives from the LDR and counted LUX. For decay measurement the measurement logic maximum light gives maximum LUX value and minimum light gives minimum LUX.

(f) Light control section: This section consists of another $5 \mathrm{~V}$ relay to control hardware such an LED to glow at a particular voltage indicating darkness as set by the user.

(g) Hardware controlling: Simple push buttons are used to start the system as well as to give the time of data transfer to the PC

(h) Data transfer: Displayed data of light intensity are transferred to RS 232 which is interfaced with microcontroller through MAX232 [12,13].

Figure 3 shows the Embedded Control Hardware Circuit with serial communicator RS232

\subsubsection{Software Description}

Software development for the project consists of two main modules one being the online monitoring and controlling and other offline analysis based on data stored in computer. Software is developed in both $\mathrm{C}$ and Assembly language. The flowchart for the program is shown in Figure 4. The value of lux is calculated using the following equation.

$$
\begin{gathered}
\text { Vout }=\text { adc_read }() * 0.0048828125 \\
\text { Lux }=500 /((10.7639 *[(5-\text { Vout })]) / \text { Vout }) \\
\text { RLDR }=[10000 *(5-\text { Vout })] / \text { Vout }
\end{gathered}
$$

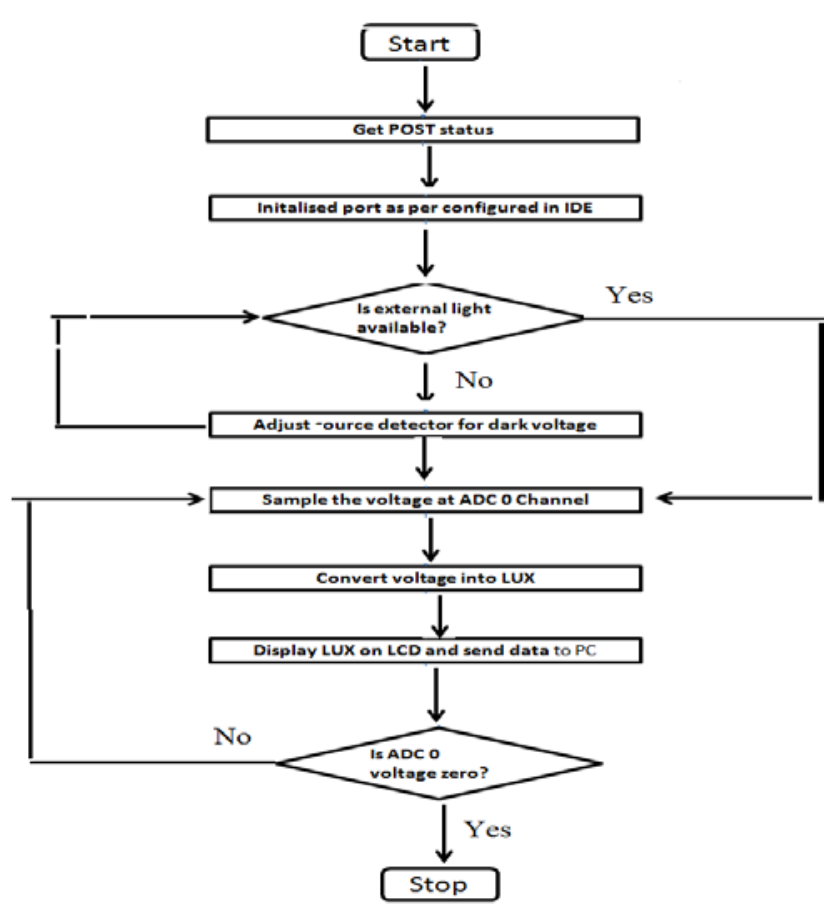

Figure 4. Controlled program flowchart

\subsection{Calibration}

The photodiode, a $10 \times 10 \mathrm{~mm}^{2}$ planar silicon cell coated with synthetic quartz is used. The photodiode is mounted behind a precisely measured 7.98-mm diameter circular aperture, yielding an active area of $0.5 \mathrm{~cm}^{2}$. The responsively is usually given every 5 nanometers. The 
calibration labs use this transfer standard to calibrate their inter-comparison working standards using a monochromatic light source. These working standards are typically identical to the equipment that will be calibrated [15].

Detectors are most often calibrated at the peak voltage at the minimum calibration check of the detector combination using identical biasing registers for the intended application. The key to this calibration transfer is a reliable kinematic mount that allows exchangeability of PIN detectors in place of LDR in the optical path, and a stable, power regulated light source. Here the calibration process is carry out with the reference of change in resistance against fixed biased resistor which is $3.3 \mathrm{k}$ $[14,15]$.

$$
\begin{aligned}
& \mathrm{RL}=500 / \mathrm{LUX} \\
& \mathrm{V}_{0}=5 *(\mathrm{RL} /(\mathrm{RL}+3.3 \mathrm{k})) \\
& \mathrm{LUX}=\left(2500 / \mathrm{V}_{0}-500\right) / 3.3 \mathrm{k}
\end{aligned}
$$

\section{Performance, Evaluation and Testing}

The system for measuring decay was verified by varying the intensity of LED using potentiometer and measuring the intensity in LUX with respect to time. The decay measurement using three types of LEDs (5W, $1 \mathrm{~W}$ and low grade LED) were also done. Table 1 shows the respective intensity with reference to time after the LED is turned off. This data is also represented on graph as shown

\begin{tabular}{|c|c|c|c|c|c|}
\hline Time in (ms) & 5W LED & 1 watt LED & Low grade LED & Accuracy Photometer 5w LED & Accuracy \%(percent) \\
\hline $6 \mathrm{~ms}$ & 5200 & 2000 & 200 & 5100.54 & 1.03 \\
\hline $12 \mathrm{~ms}$ & 2800 & 1930 & 193 & 2775.77 & 0.8653 \\
\hline $18 \mathrm{~ms}$ & 1500 & 1560 & 156 & 1485.60 & 0.96 \\
\hline $24 \mathrm{~ms}$ & 830 & 1370 & 137 & 830.22 & 0.0265 \\
\hline $30 \mathrm{~ms}$ & 750 & 1230 & 123 & 750.56 & 0.07466 \\
\hline $36 \mathrm{~ms}$ & 700 & 1130 & 113 & 700.23 & 0.032 \\
\hline $42 \mathrm{~ms}$ & 642 & 1023 & 102.3 & 642.33 & 0.0514 \\
\hline $48 \mathrm{~ms}$ & 570 & 950 & 95 & 570.45 & 0.07894 \\
\hline $54 \mathrm{~ms}$ & 523 & 725 & 72.5 & 523.22 & 0.0420 \\
\hline $60 \mathrm{~ms}$ & 435 & 460 & 46 & 435.86 & 0.1977 \\
\hline $66 \mathrm{~ms}$ & 370 & 320 & 32 & 370.22 & 0.0594 \\
\hline $72 \mathrm{~ms}$ & 290 & 241 & 24.1 & 290.11 & 0.03793 \\
\hline $78 \mathrm{~ms}$ & 168 & 115 & 11.5 & 168.33 & 0.1964 \\
\hline $84 \mathrm{~ms}$ & 130 & 75 & 7.5 & 130.44 & 0.3384 \\
\hline $90 \mathrm{~ms}$ & 85 & 42 & 4.2 & 85.33 & 0.3882 \\
\hline $96 \mathrm{~ms}$ & 57 & 35 & 3.5 & 57.05 & 0.08771 \\
\hline $103 \mathrm{~ms}$ & 39 & 15 & 1.5 & 39.65 & 1.666 \\
\hline $109 \mathrm{~ms}$ & 21 & 9 & 0.9 & 21.45 & 2.14 \\
\hline $115 \mathrm{~ms}$ & 16 & 5 & 0.5 & 16.66 & 4.125 \\
\hline $121 \mathrm{~ms}$ & 9 & 5 & 0.5 & 9.33 & 3.666 \\
\hline $127 \mathrm{~ms}$ & 5 & 5 & 0.5 & 5.66 & 13.3 \\
\hline
\end{tabular}
in Figure 5.

Table 1. Intensity of LED's (5W, 1W and low grade) with respect to time.

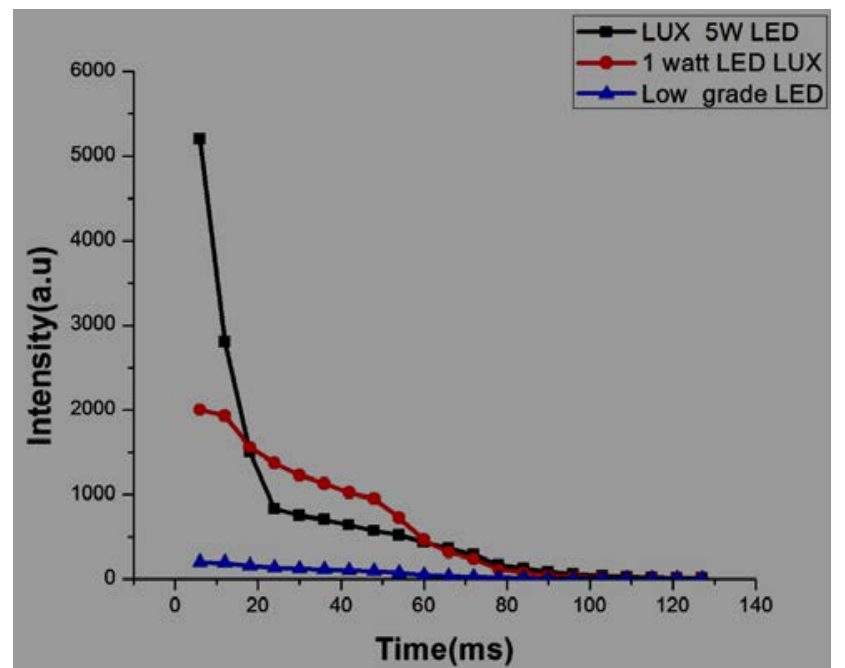

Figure 5. Decay measurement w.r.t. time

\section{Conclusion}

The system to measure the decay time is successfully designed and is giving satisfactory results with the average accuracy of 1.3845 LUX measured with the standard photometer. This low cost, simple system can be used to measure the decay of luminescence in phosphors used in display. The results observed from ta Table 1 shows that as time increases the accuracy of the system is decreases as bit resolution voltage increases from $625 \mathrm{mV} / \mathrm{Bit}$ to $1.38 \mathrm{~V} / \mathrm{Bit}$.

\section{Acknowledgement}

One of the author VVR is grateful to UGC, New Delhi for sanctioning the leave under FIP to carry out this research work. The author will thankful to the Department of Electronics for providing the lab facility for Development of system also thankful to Department physics, Luminescence Research Group for photometer measurement

\section{References}

[1] J.P. Boeuf, J. Phys. D 36 (2003) R53.

[2] Y.F. Wang, X. Xu, L.J. Yin, L.Y. Hao, Electrochemical. Solid State Letts. 13 (2010) J119.

[3] K.Y. Jung, H.W. Lee, Y.C. Kang, S.B. Park, Y.S. Yang, Chem. Mater. 17 (2005) 2729.

[4] D.S. Zang, J.H. Song, D.H. Park, Y.C. Kim, D.H. Yoon, J. Lumina. 129 (2009) 1088.

[5] D.V O'Connor, D. Phillips, Time-Correlated Single Photon Counting, Academic Press, 1984. 
[6] Muhammad Ali Mazidi and Janice Gillispe Mazidi, “The 8051 microcontroller and embedded systems", Pearson education ltd. Indian edition first, Dorling Kindersley india Pvt.Ltd India, 2013.

[7] www.ni.com/dataloggers - "A Review of PC -Based Data Logging and Recording Techniques” National Instrument, India, 2008.

[8] Muhammad Ali Mazidi and Janice Gillispe Mazidi, "The 8051 microcontroller and embedded systems", Pearson education ltd. Indian edition first, Dorling Kindersley India Pvt. Ltd India, 2013.

[9] National Semiconductor Corporation, "ADC 0809 data sheet-8-bit Microprocessor compatible A/D converters with 8-channel multiplexer", national Semiconductor data book, 2002.
[10] National Semiconductor Corporation, "precision centigrade temperature sensors", Atmel data book, 2000.

[11] Atmel Corporation, "8-bit microcontroller with 8k bytes flash", Atmel Data book, 2000.

[12] H S kalsi, "Electronic instrumentation", Tata McGraw-Hill Ltd., New Delhi, 1999.

[13] H. Szmacinski and Q. Chang, “Appl. Spectroscope.” 106,2000.

[14] B. Yuan, B. S. R. McClellan, B. F. Al-Mifgai, E. A. Growney, “Am. J. Phys." 78, 2010.

[15] J.Chen, "Ruthenium (II) pyrene-bipyridine complexes: Synthesis, photo physics, photochemistry and in vivo oxygen sensing," "Ph.D. Thesis", Tulane University, 2010. 